\title{
An Unusual Case of Klebsiella pneumoniae Endocarditis
}

\author{
Syed Adeel Hassan ${ }^{1}$, Ali Akhtar ${ }^{2}$, Noor Ul Falah ${ }^{3}$, Maham Khan ${ }^{4}$ \\ 1. Internal Medicine, Dow University of Health Sciences, Karachi, PAK 2. Internal Medicine, Army Medical College, \\ National University of Medical Sciences, Rawalpindi, PAK 3. Internal Medicine, King Edward Medical University, \\ Lahore, PAK 4. Radiology, Armed Forces Institute of Radiology and Imaging, Rawalpindi, PAK
}

Corresponding author: Syed Adeel Hassan, doc.syed92@gmail.com

\begin{abstract}
Klebsiella pneumoniae notoriously causes life-threatening community-acquired or hospital-acquired pneumonia. In the United States, community-acquired pneumonia is a relatively common diagnosis. However, community-acquired pneumonia due to Klebsiella pneumoniae is fairly uncommon. Delayed antibiotic administration can result in bacteremia, septicemia and other systemic complications. Infective endocarditis arising as a complication of community-acquired Klebsiella pneumoniae infection has rarely been reported. Our patient is an 88-year-old diabetic female, who was admitted to our intensive care unit due to a high-grade fever, worsening dyspnea and hypotension. Chest $\mathrm{x}$-ray and blood culture were conclusive for pneumonia due to Klebsiella pneumoniae. Importantly, the species was only susceptible to colistin. Furthermore, an echocardiogram revealed mobile vegetations of the non-coronary cusp of the aortic valve. Treatment with colistin resulted in clinical improvement and an uneventful discharge from the hospital. Follow up echocardiography was scheduled upon discharge to monitor the resolution of cardiac vegetations.
\end{abstract}

Categories: Cardiology, Internal Medicine, Infectious Disease

Keywords: klebsiella pneumoniae, endocarditis, pneumonia, colistin, aortic valve

\section{Introduction}

Klebsiella pneumoniae is a gram-negative bacteria belonging to the Enterobacteriaceae family. Carl Friedlander first isolated the bacterium from the lungs of patients who had died from pneumonia. Alcoholic and diabetic patients are at an increased risk of infection. The bacteria colonize the mucosal surfaces of the oropharynx and gastrointestinal tract. Higher rates of colonization have been reported in chronic alcoholics. Pneumonia caused by the bacteria can be broken down into hospital-acquired or community-acquired infections. Klebsiella pneumoniae accounts for 3\%-5\% of all community-acquired pneumonia cases. It is also known to be the cause in approximately $11.8 \%$ of cases in hospital-acquired pneumonia [1]. However, pneumonia and endocarditis due to Klebsiella pneumoniae infection are extremely rare [2]. We report a case of community-acquired Klebsiella pneumoniae infection which presented initially with pneumonia. It then clinically progressed to bacteremia resulting in sepsis. The bacteremia further involved the aortic valve resulting in endocarditis.

Received 02/09/2020

02/12/2020 Review ended 02/14/2020 Published 02/14/2020

\section{Copyright 2020}

Hassan et al. This is an open access article distributed under the terms of the Creative Commons Attribution License CC-BY 4.0., which permits unrestricted use, distribution, and reproduction in any medium, provided the original author and source are credited.

\section{Case Presentation}

An 88-year-old female was admitted to our medical intensive care unit with a two-day history of worsening dyspnea and hypotension. An initial assessment at the time of admission ruled out pulmonary embolism. On further inquiry, the patient had a history of consistent, unremitting, high-grade fever $\left(100^{\circ} \mathrm{F}\right)$ for the past week. It was also noted that her dyspnea developed after the onset of high-grade fever. Additionally, she had also developed a mildly productive cough during the past week. Her past medical history was significant for long-standing uncontrolled diabetes and end-stage renal disease. Family members of the patient declined any history of bird exposure at home or as part of her occupation. Social history revealed that she was a nonsmoker and non-alcoholic. Furthermore, she did not have any history of illicit drug abuse.

A few hours after her admission, her condition began to deteriorate. Her oxygen saturation dropped from $89 \%$ to $70 \%$. She became severely dyspneic with a raised blood $\mathrm{PaCO}_{2}$ level. Based on the rapid clinical decline, the decision was taken to electively intubate her and further proceed with treatment on the grounds of sepsis. On physical examination, she was febrile $\left(100^{\circ} \mathrm{F}\right)$, with blood pressure $80 / 50 \mathrm{mmHg}$ and pulse 88 beats/min. Respiratory system examination was significant for bilateral basal crepitations with an expiratory wheeze. Chest $\mathrm{x}$-ray revealed patchy areas of alveolar opacification in bilateral lung fields. Opacification predominantly involved the right upper and middle lung fields and left upper and lower lung fields. The diseased regions in the right lung zones collapsed to form a non-homogeneous wedge-shaped area of opacification with internal air bronchograms (Figure 1). Furthermore, physiological calcification of aortic knuckle was also evident. Based on the chest x-ray findings, a preliminary diagnosis of pneumonia was made. 


\section{Cureus}

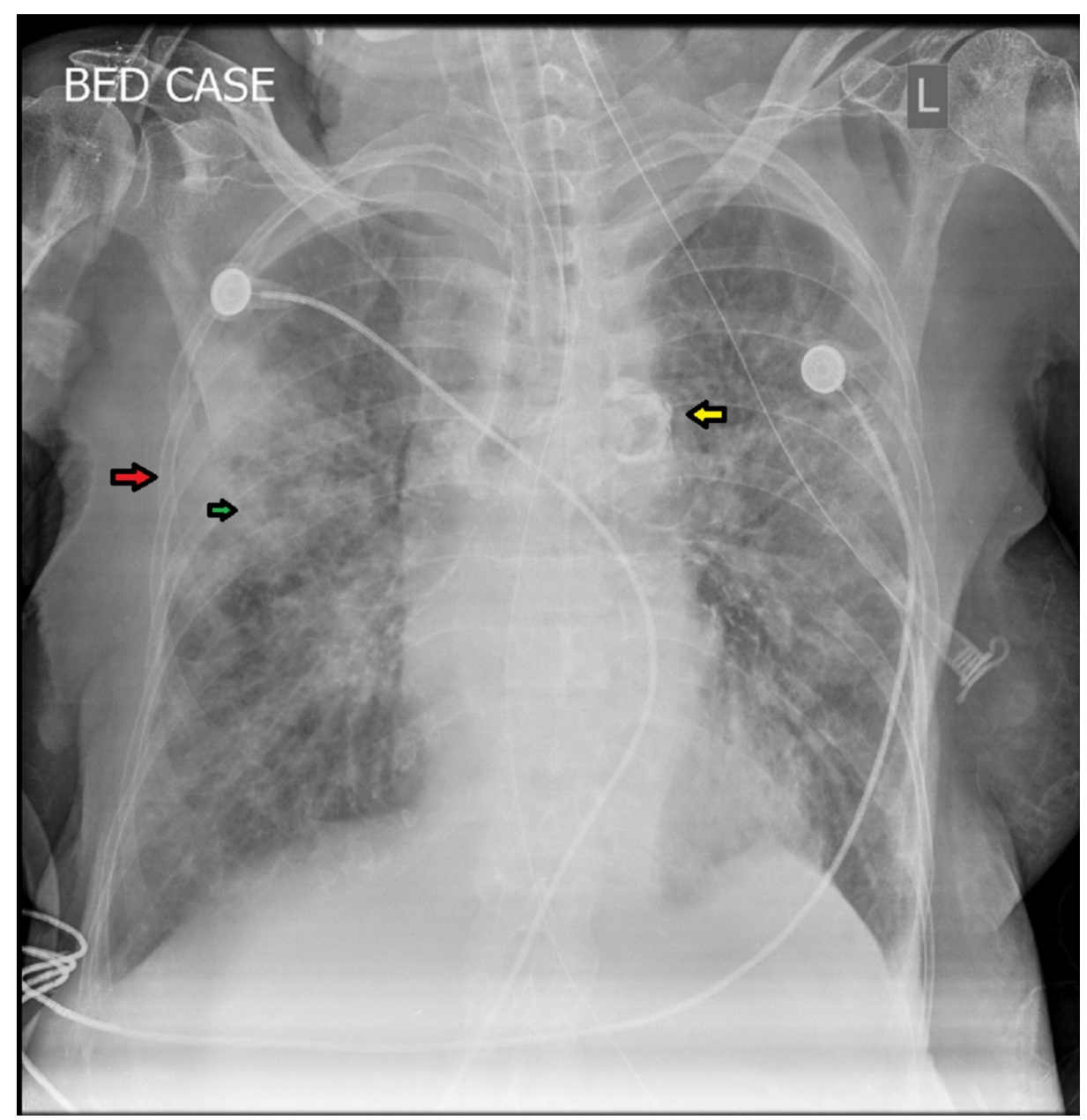

FIGURE 1: Chest X-ray depicting pathological changes of pneumonia in our patient

L: Left-sided orientation of the patient

Bed case: Bedside chest X-ray

Widespread opacification of the respective right and left lung fields can be observed. The right upper and middle lung zone opacities have fused to impart a wedge-shaped area of opacification (red arrow). Within this wedge-shaped area, dark air-filled bronchi are being made visible by the surrounding opacification of alveoli (air bronchogram, green arrow). Physiological calcification of the aortic knuckle can also be noted (yellow arrow).

An echocardiogram revealed no obvious clots in the left ventricle. However, it was significant for mobile vegetations on the non-coronary cusp of the aortic valve (Figure 2). A grade I diastolic dysfunction with a global ejection fraction of $58 \%$ was also noted. 


\section{Cureus}

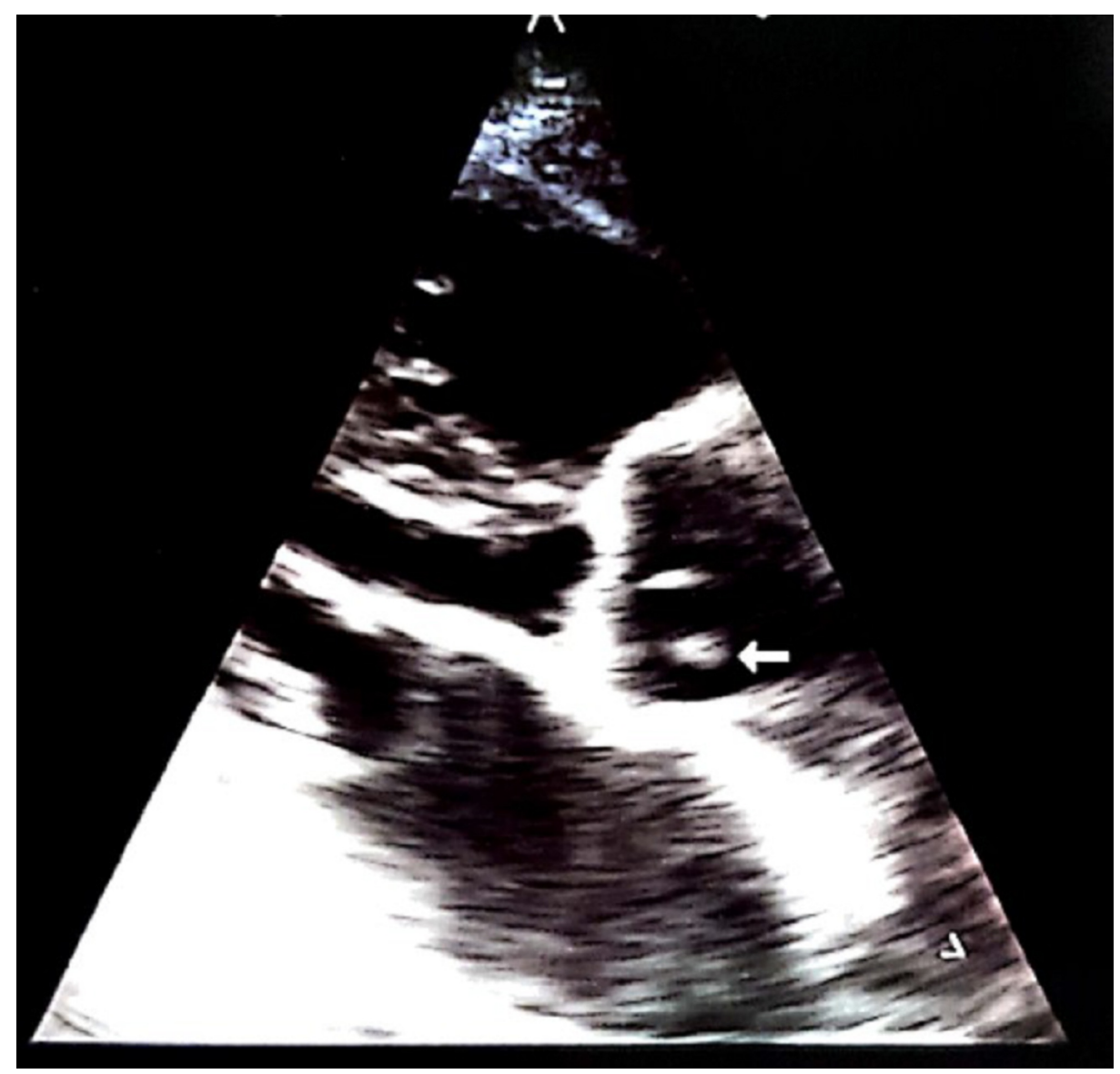

FIGURE 2: Patient's echocardiogram depicting valvular vegetations

Mobile vegetations on the non-coronary cusp of the aortic valve can be seen (white arrow).

On the second day of admission, she suffered from a higher fever spike $\left(102^{\circ} \mathrm{F}\right)$. This bout of fever was accompanied by leukocytosis (total leukocyte count $26.8 \times 10^{9} / \mathrm{L}$ with a neutrophil count of $92 \%$ ). The patient was started on broad-spectrum antibiotics vancomycin and cefepime. Subsequently, the result of cough sputum culture was negative. However, blood culture was positive with Klebsiella pneumoniae species which was only susceptible to colistin. The antibiotic was changed to colistin and was continued for 10 days. Thereafter, her clinical condition improved. Follow-up echocardiography was scheduled upon discharge to monitor the resolution of the vegetations.

\section{Discussion}

Studies have identified clinical differences between hospital-acquired and community-acquired Klebsiella pneumoniae infections [3]. Community-acquired infections present initially with septic shock and respiratory failure [2]. It can also present with rapid onset fever and hemoptysis. These infections are rare in North America, Western Europe and Australia. It accounts for less than $1 \%$ of pneumonia cases requiring hospitalization [3]. The organism has the propensity to cause disease of the chest, urinary and intraabdominal systems [4]. However, cases of bacteremia due to Klebsiella pneumoniae remain prevalent in Asia and Africa [3,5].

Klebsiella infections can metastasize to other organs and result in septic complications. Urinary tract infections and liver abscess are the most common sources of infection in Klebsiella pneumoniae bacteremia [6]. The portal of infection entry is only identified in $32 \%$ of cases [4]. Other infection entry routes such as vascular, oral or lung have rarely been reported [4]. Predisposing factors for infection include liver cirrhosis, hepatobiliary diseases, diabetes mellitus, asplenia, neoplasia, chronic alcoholism and corticosteroids $[4,6]$. In patients with community-acquired Klebsiella infections, diabetes mellitus is the most commonly reported predisposing factor [6]. Endocarditis due to Klebsiella pneumoniae infection accounts for $5 \%$ of all cases of endocarditis [5]. A review of 50 cases indicated that the aortic valve is most commonly involved, followed by the mitral valve $[2,5]$. The mortality rate for such cases is more than $50 \%[5]$. The mitral valve tends to be involved when bacteremia due to a pyogenic liver abscess tends to predominate [2]. Significant risk factors for mortality include septic shock, respiratory failure, lung infection, leukopenia, thrombocytopenia and inappropriate antimicrobial therapy $[2,6]$. 
Consistent with community-acquired Klebsiella pneumoniae infections, our patient presented with a toxic clinical presentation comprising of respiratory difficulty and septic shock-like presentation. The initial fulminant pneumonia likely acted as a source of progression to bacteremia. This resulted in clinical deterioration due to the development of septic shock. Pneumonia was also a nidus for the development of aortic valve vegetation in our case. Also consistent was the presence of long-standing diabetes mellitus as a predisposing risk factor and aortic valve involvement. As opposed to the findings of high mortality in literature, our patient had a good clinical outcome with colistin. A delay in seeking medical care and the administration of appropriate antibiotics could be responsible for the rapid clinical progression to septic shock and the development of endocarditis.

\section{Conclusions}

Community-acquired Klebsiella pneumoniae infections seldom occur. However, cases of bacteremia are still common in Asia. Pneumonia can act as a source of clinical progression to bacteremia. As a consequence, complications such as septic shock and endocarditis ensue. In community-acquired cases, the aortic valve tends to be the most commonly involved heart valve. Commonly associated predisposing factors for infection include chronic alcoholism and diabetes mellitus. This case highlights the severity and clinical consequences of community-acquired Klebsiella pneumoniae infections. It also indicates the need for the early management of the patient to avoid the development of bacteremic clinical consequences.

\section{Additional Information}

\section{Disclosures}

Human subjects: Consent was obtained by all participants in this study. Conflicts of interest: In compliance with the ICMJE uniform disclosure form, all authors declare the following: Payment/services info: All authors have declared that no financial support was received from any organization for the submitted work. Financial relationships: All authors have declared that they have no financial relationships at present or within the previous three years with any organizations that might have an interest in the submitted work. Other relationships: All authors have declared that there are no other relationships or activities that could appear to have influenced the submitted work.

\section{References}

1. Klebsiella pneumonia. (2019). Accessed: January 5, 2020: https://www.ncbi.nlm.nih.gov/books/NBK519004/.

2. Hwang JA, Her C, Kim YW: Endocarditis caused by community-acquired Klebsiella pneumoniae infection . Korean J Crit Care Med. 2013, 28:41-45. 10.4266/kjccm.2013.28.1.41

3. Klebsiella species (K. pneumoniae, K. oxytoca, K. ozaenae and K. rhinoscleromatis). (2016). Accessed: January 6, 2020: http://antimicrobe.org/new/b107.asp.

4. Fane ML, Dollo I, Sodqi M, et al.: Septicaemia caused by community acquired Klebsiella pneumonia complicated with endocarditis and meningoencephalitis: a case report. J Cardiovasc Dis Diagn. 2016, 4:244. 10.4172/2329-9517.1000244

5. Rivero A, Gomez E, Alland D, Huang DB, Chiang T: K2 serotype Klebsiella pneumoniae causing a liver abscess associated with infective endocarditis. J Clin Microbiol. 2010, 48:639-641. 10.1128/JCM.01779-09

6. Tsay RW, Siu LK, Fung CP, Chang FY: Characteristics of bacteremia between community-acquired and nosocomial Klebsiella pneumoniae infection: risk factor for mortality and the impact of capsular serotypes as a herald for community-acquired infection. Arch Intern Med. 2002, 162:1021-1027.

10.1001/archinte.162.9.1021 